\title{
Desenvolvimento de juvenis de Piracanjuba (Brycon orbignyannus), Vallencienes (1849) (Teleostei: characidae) em tanques experimentais fertilizados com adubação orgânica
}

\author{
Development of fingerlings of Piracanjuba (Brycon orbignyannus), \\ Vallencienes (1849) (Teleostei: characidae) in tanks fertilized with \\ organic manures
}

\author{
Aldi Feiden ${ }^{1 *}$; Carmino Hayashi ${ }^{2}$
}

Resumo

O objetivo deste trabalho foi estudar o desenvolvimento de juvenis de Piracanjuba (Brycon orbignyanus), em tanques com adubações orgânicas. O experimento, inteiramente casualizado, foi realizado em 16 tanques de 1000 litros, adubados com estercos de bovinos (BOV), suínos (SUI), frangos de corte (FRG) e outros sem adubação (SAO), utilizando 15 peixes $/ \mathrm{m}^{3}$ com peso e comprimento médios iniciais de $10,87 \pm 0,31 \mathrm{~g} \mathrm{e} 9,78 \pm 0,07 \mathrm{~cm}$. Após 30 dias, o experimento mostrou um desenvolvimento uniforme dos peixes e alta taxa de sobrevivência na densidade estudada. Os tratamentos apresentaram diferenças estatisticamente significativas $(\mathrm{P}<0,05)$ para ganho de peso e crescimento diário, com exceção dos tratamentos BOV e SUI quando comparados entre si. A temperatura e o oxigênio dissolvido foram os fatores abióticos que exerceram maior influência sobre a biota aquática, afetando o desenvolvimento dos peixes. Entre o fitoplâncton houve maior abundância de organismos nanoplanctônicos favorecendo o desenvolvimento do zooplâncton, destacando-se as clorófitas, com o gênero Scenedesmus, e as cianofíceas, com o gênero Microcystis, e entre o zooplâncton, a maior abundância foi de rotíferos dos gêneros Brachionus e Keratella, seguido por copépodas. O tratamento adubado com esterco de frangos possibilitou um maior desenvolvimento da comunidade planctônica, e melhores resultados quanto ao desenvolvimento dos peixes, demonstrando a importância do alimento natural na sua dieta.

Palavras-chave: Alimentação, Brycon orbignyanus, plâncton, fertilização de tanques

\begin{abstract}
The objective of this paper was to study the development of Piracanjuba (Brycon orbignyanus) in tanks under differents organic fertilizers. The experiment, entirely randomized, was accomplished in 16 tanks of 1000 liters, fertilized with manures of bovine (BOV), pigs (SUI), chickens (FRG) and others without fertilizer (SAO), using $15 \mathrm{fish} / \mathrm{m}^{3}$ with an initial average weight and length of $10,87 \pm 0,31 \mathrm{~g} \mathrm{e} 9,78 \pm 0,07 \mathrm{~cm}$. After 30 days, the experiment showed a uniform development of the fish and high survival rate in the studied density. The treatments presented significant statistical differences $(\mathrm{P}<0,05)$ for weight gain and daily growth, except for the treatments BOV and SUI when compared to each other. The temperature and
\end{abstract}

1 Engenheiro Agrônomo, Professor Adjunto - Universidade Estadual do Oeste do Paraná - Campus de Toledo - UNIOESTE Toledo/PR. E-mail:aldi@unioeste.br.

2 Biólogo - Prof. Titular, Programa de Pós-Graduação em Ecologia de Ambientes Aquáticos Continentais - Universidade Estadual de Maringá. PEA-UEM. E-mail: chayashi@ netsite.com.br

* Autor para correspondência. 
the dissolved oxygen were the abiotic factors that exerced a larger influence on the aquatic biota, affecting the development of the fish. Among the phytoplankton there was a larger presence of nanoplanktonic organisms favoring the development of the zooplankton, mainly the Clorophyta, with the Scenedesmus species, and the Cianophyta, with the Microcystis species, and among the zooplankton, the largest presence was of the rotifera from the species Brachionus and Keratella, followed by the copepods. The treatment fertilized with manure of chickens made possible a larger development of the plankton community, and better results of the development of the fish, demonstrating the importance of natural food in its diet.

Key words: Feeding, Brycon orbignyanus, plankton, pond fertilizing

\section{Introdução}

Os estudos sobre os peixes do gênero Brycon iniciaram-se em 1927 com Rodolpho von Ihering, mas até hoje não são conhecidos muitos aspectos de sua biologia. Segundo Britski, Sato e Rosa (1988) e Ceccarelli e Senhorini (1996), existem cerca de 40 espécies classificadas e quanto a sua classificação, estes peixes pertencem à Superordem Ostariophysi, Ordem Characiformes, Família Characidae, Subfamília Bryconinae e Gênero Brycon.

Os peixes do gênero Brycon distribuem-se pelas principais bacias hidrográficas brasileiras, sendo que na bacia Amazônica ocorre o matrinchã ( $B$. cephalus), na bacia do rio Paraná a piracanjuba ( $B$. orbignyanus), na bacia do rio São Francisco o matrinchã (B. lundii), na bacia do rio ParaguaiPantanal a piraputanga (B. hilarii), e na bacia do Leste (rio Paraíba do Sul) a piabanha (B. insignis), a Pirapitinga do Sul (B. reinhardti), e as espécies (B. devillei) e (B. pesu), conforme Paiva (1983) e Mendonça (1996). Considerados grandes migradores, os briconídeos são peixes reofílicos e apresentam maturidade sexual aos três anos de idade (CECCARELLI; SENHORINI, 1996), possuindo desova total com ovos livres e sem cuidados com a prole (NAKATANI et al., 2001).

Apresentam um grande potencial para a aqüicultura e podem ser utilizados para repovoamento ou reforços de populações em rios onde estejam ameaçados de extinção. A Piracanjuba (B. orbignyanus) é uma espécie nativa da bacia do rio Paraná, onde em décadas passadas podia ser encontrada com abundância.
Muito sensível às degradações ambientais e superexplotada pela pesca (COMPANHIA DE TECNOLOGIA DE SANEAMENTO AMBIENTAL, 1982), hoje é raro o encontro desta espécie em ambientes naturais. Segundo Paiva apud Conte, Bosano e Ferraz de Lima (1995), B. orbignyanus é uma das espécies mais prejudicadas com a construção de barragens nos rios, estando sujeito à extinção. Além das barragens, o desmatamento das áreas marginais dos rios através da expansão agrícola, contribuiu para a diminuição dos estoques. Conforme Universidade Estadual de Maringá (1989) e Universidade Estadual de Maringá (1993), na região do Alto Rio Paraná, a freqüência relativa de captura e a biomassa relativa de $B$. orbignyanus é menor que $1 \%$, tendo sido amostrada no rio Paraná, seus afluentes, e nas lagoas marginais. Agostinho e Zalewski (1996) afirmam que a presença de adultos de $B$. orbignyanus na época de desova nos afluentes do rio Paraná na área da planície de inundação, confirma a tese de que os represamentos causam um grande impacto sobre a espécie.

As pesquisas sobre $B$. orbignyanus concentraram-se até hoje basicamente em sua taxonomia, reprodução e poucos estudos foram realizados sobre o seu comportamento alimentar. A espécie apresenta no ambiente natural uma alimentação baseada na coleta de pequenos frutos e sementes (MENDONÇA, 1994), e na fase larval e jovem alimenta-se de zooplâncton e de larvas de peixes. Mendonça (1996) declara que na fase larval, o B. orbignyanus apresenta alto canibalismo, o qual 
é iniciado poucas horas após a eclosão, sendo hoje a principal limitação tecnológica para a obtenção de indivíduos jovens. Os conhecimentos de seu comportamento e preferências alimentares são fundamentais para que se possam desenvolver tecnologias para sua criação, tanto para fins econômicos como para repovoamentos em rios e represas, pois a utilização de juvenis proporciona uma maior possibilidade de sobrevivência, devido a maiores chances de escape de predadores.

Os organismos planctônicos são muito importantes na alimentação dos estágios de desenvolvimento iniciais dos peixes, e a sua disponibilidade no ambiente pode levar a um melhor desempenho em relação ao desenvolvimento. A preferência alimentar varia de espécie para espécie, de acordo com o desenvolvimento de seus órgãos e com a capacidade dos indivíduos jovens de aceitar alimentos inertes.

A produção planctônica pode ser aumentada com o uso de adubos orgânicos e inorgânicos e sua importância na produção de organismos planctônicos para alimentação de peixes é destacada nos trabalhos de Boyd (1982) e Hepher (1988), sendo que no Brasil podemos citar alguns experimentos realizados com utilização de adubos orgânicos, como Paranaguá e Koening (1980), Oliveira e Silva et al. (1984), Motokubo et al. (1988), Senhorini et al. (1988), Ferrari, Lucas e Gaspar (1991), Doria et al. (1993), Doria e Leonhardt (1993), Faria (1997), Ribeiro, Kubo e Mainardes-Pinto (1997) e Soares et al. (1997).

O objetivo deste trabalho foi ampliar os conhecimentos sobre a biologia de B. orbignyanus e obter subsídios sobre a fase mais crítica de desenvolvimento que é a fase inicial, testar a influência de diferentes tipos de adubos orgânicos na produção planctônica e a disponibilidade dos diferentes organismos planctônicos para a alimentação dos alevinos da espécie, e estudar as relações existentes entre os fatores abióticos e bióticos da água, além de avaliar o incremento de biomassa, a conversão alimentar aparente e a taxa de sobrevivência.

\section{Materiais e Métodos}

O estudo consistiu na instalação de um experimento com indivíduos jovens de $B$. orbignyanus, em janeiro de 1998 com duração de 30 dias, em tanques do Laboratório de Aqüicultura do Departamento de Biologia da Universidade Estadual de Maringá. Foram utilizados 16 tanques com capacidade de 1.000 litros, utilizando-se um total de 240 indivíduos com peso e comprimento médio

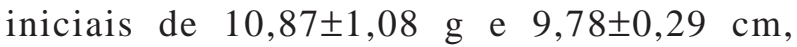
respectivamente, com densidade de estocagem inicial de 15 peixes $/ \mathrm{m}^{3}$.

O delineamento experimental utilizado foi inteiramente casualizado, com quatro tratamentos e quatro repetições, sendo que os tratamentos tiveram diferentes substratos: BOV-Adubação com esterco de bovinos; SUI-Adubação com esterco de suínos; FRG-Adubação com esterco de frangos de corte; e SAO-Sem adubação orgânica. Os tanques foram inoculados com "plâncton selvagem" sete dias antes da estocagem, e a adubação inicial foi de $30 \mathrm{~g} / \mathrm{m}^{3}$ de adubo orgânico seco, finamente triturado, quando da inoculação, e seguida por adubações semanais com $15 \mathrm{~g} / \mathrm{m}^{3}$ nas duas primeiras semanas. A renovação da água dos tanques foi de $5 \%$ ao dia. A alimentação baseou-se no fornecimento de ração comercial com $28 \%$ de proteína bruta, na forma de ração extrusada, fornecida uma vez ao dia, pela manhã, durante todos os dias da semana, na proporção inicial de $8 \%$ da biomassa, sendo reduzida a $4 \%$ ou suspensa quando haviam sobras. A ração foi pesada em balança semianalítica com duas casas decimais.

Durante o período experimental foram realizadas quatro biometrias $(7,14,22$ e 30 dias após o início do experimento), retirando todos os indivíduos para pesagem e medição do comprimento total, através de balança com precisão de $0,1 \mathrm{~g}$ e ictiômetro graduado em milímetros. O desenvolvimento foi determinado através do Índice de Crescimento Específico de Ricker (Ferraz de Lima et al., 1988), e através do Índice de Conversão Alimentar Aparente (ICA) de acordo com Steffens (1987). A análise 
estatística para o peso médio e para ganho diário de peso foi feita pela análise de variância e pelo teste de Tukey para detectar diferenças entre os tratamentos, com base em Pimentel (1987) e Koehler (1994). A qualidade de água foi analisada através da tomada dos parâmetros físicos e químicos e foram monitorados diariamente a temperatura da água, o $\mathrm{pH}$, a condutividade elétrica $(\mathrm{S} / \mathrm{cm})$, e oxigênio dissolvido (mg/L), às 9:00 e 16:00 hortas.

Diariamente foram retiradas amostras para análise do plâncton, realizando-se a coleta de 5 amostras de $250 \mathrm{~mL}$ em diferentes pontos dos tanques, que foram homogeneizadas, formando uma amostra final de $250 \mathrm{~mL}$, filtrada em rede de malha $20 \mathrm{~m}$, e fixada em formol $4 \%$, e feita a análise quantitativa e qualitativa do fito e zooplâncton sob microscópio estereoscópio. A identificação do fitoplâncton foi subsidiada pelos trabalhos de Wetzel (1981), Needham e Needham (1982), PicelliVicentim (1987), Xavier (1993), Huszar, Werneck e Esteves (1994) e Oliveira, Train e Rodrigues (1994), e a identificação do zooplâncton baseou-se nos trabalhos de Lewis Jr (1979), Needham e Needham (1982), Sendacz e Kubo (1982), Matsumura-Tundisi e Rocha (1983) e Bonecker, Lansac-Tôha e Staub (1994).

\section{Resultados e Discussão}

Os dados referentes ao desenvolvimento de $B$. orbignyanus (Tabela 1 e Figura 1) mostram que o tratamento FRG obteve resultados melhores que os demais, seguido pelos tratamentos BOV e SUI e SAO. A conversão alimentar média dos tratamentos do período estudado variou entre 1,49 e 1,94, estando de acordo com trabalho de Conte, Bozano e Ferraz de Lima (1995), os quais afirmam que alta densidade tem influência estimulante no comportamento alimentar da espécie. Borghetti, Canzi e Nogueira (1991) comprovaram que o teor de proteína de $40 \%$ obteve o melhor resultado em tanques-rede para peixes em crescimento, mostrando a alta exigência do alimento protéico para a conversão alimentar e o desenvolvimento da espécie.

Tabela 1. Resultados do desenvolvimento de piracanjuba (Brycon orbignyanus) durante 30 dias de experimento em tanques experimentais, fertilizados com diferentes adubos orgânicos

\begin{tabular}{lcccc}
\hline \multicolumn{1}{c}{ Parâmetros|Tratamentos } & BOV & SUI & FRG & SAO \\
\hline Sobrevivência (\%) & 100,00 & 95,00 & 96,67 & 98,33 \\
Tamanho médio inicial (cm) & $9,78 \pm 0,07$ & $9,78 \pm 0,07$ & $9,78 \pm 0,07$ & $9,78 \pm 0,07$ \\
Peso inicial (g) & $10,87 \pm 0,31$ & $10,87 \pm 0,31$ & $10,87 \pm 0,31$ & $10,87 \pm 0,31$ \\
Tamanho médio final (cm) & $14,15 \pm 0,25$ & $14,05 \pm 0,22$ & $14,49 \pm 0,12$ & $13,92 \pm 0,17$ \\
Peso final (g) & $496,30 \mathrm{~b}$ & $485,47 \mathrm{~b}$ & $543,26 \mathrm{a}$ & $454,94 \mathrm{c}$ \\
Ração fornecida (g) & 564,5 & 565,5 & 559,2 & 499,9 \\
Crescimento diário (g/dia) & 11,24 & 10,88 & 12,51 & 9,62 \\
Cresc. diário individual (g/dia) ${ }^{*}$ & $0,77 \mathrm{~b}$ & $0,78 \mathrm{~b}$ & $0,88 \mathrm{a}$ & $0,64 \mathrm{c}$ \\
Índice Cresc. Esp. de Ricker (G) & 2,77 & 2,74 & 2,52 & 3,14 \\
Índice Conversão Alim. & 1,67 & 1,73 & 1,49 & 1,94 \\
Aparente & & & & \\
\hline
\end{tabular}

"Os valores seguidos da mesma letra não diferem significativamente entre si pelo teste de Tukey.

Tratamentos: BOV - Bovinos; SUI - Suínos; FRG - Frangos; SAO - Sem adubação orgânica 


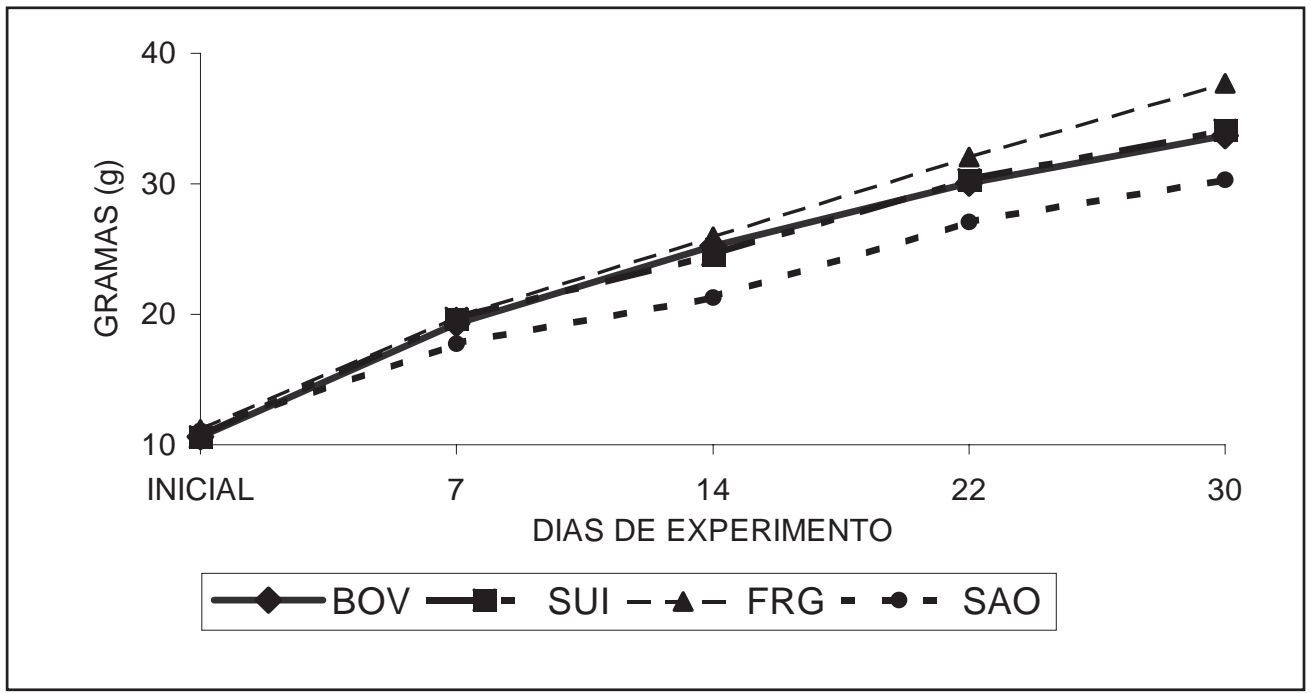

Figura 1. Peso médio dos diferentes tratamentos experimentais

Como o teor de proteína da ração fornecida foi de apenas $28 \%$ PB, a alta conversão alimentar inicial pode ser creditada ao consumo de plâncton, pois de acordo com Hepher (1988), o zooplâncton apresenta teores de proteína de 64,3\% (rotíferos), 56,5\% (cladóceros) e 52,3\% (copépodas). Os resultados referentes ao Índice de Conversão Alimentar
Aparente (ICA), apresentados na Tabela 2, mostram que a partir da segunda semana de experimento ocorreu um decréscimo da conversão alimentar. $\mathrm{Na}$ terceira semana, houve uma redução no consumo de ração, e isto levou a diminuição do fornecimento, tendo sido suspenso o fornecimento no $17^{\circ}$ dia.

Tabela 2. Índice de Conversão Alimentar Aparente dos diferentes tratamentos durante o experimento com Piracanjuba Brycon orbignyanus em tanques experimentais, fertilizados com diferentes adubos orgânicos

\begin{tabular}{cccccc}
\hline Tratamento & 1 a 7 dias & 8 a 14 dias & 15 a 22 dias & 23 a 30 dias & Média \\
\hline BOV & 0,78 & 1,13 & 1,68 & 4,49 & 1,67 \\
SUI & 0,71 & 1,44 & 1,75 & 5,09 & 1,73 \\
FRG & 0,62 & 1,18 & 1,25 & 3,73 & 1,49 \\
SAO & 0,68 & 1,66 & 1,27 & 4,62 & 1,94 \\
\hline
\end{tabular}

Tratamentos: BOV - Bovinos; SUI - Suínos; FRG - Frangos; SAO - Sem adubação orgânica

A análise de variância dos dados de peso final e crescimento diário individual apresentaram diferenças estatisticamente significativas $(\mathrm{P}<0,05)$ entre os tratamentos, com FRG apresentando o maior peso final. A aplicação do teste de Tukey revelou que os tratamentos diferem estatisticamente entre si, com exceção dos tratamentos BOV e SUI que, quando comparadas entre si, não foram estatisticamente diferentes. A taxa de sobrevivência foi alta para todos os tratamentos, não havendo diferenças significativas entre eles, mas alguns peixes mortos entre o $15^{\circ} \mathrm{e}$ o $18^{\circ}$ dia foram parcialmente devorados, demostrando que a espécie possui tendência ao canibalismo nesta idade, devido ao estresse causado pela alteração das características físico-químicas da água. Tavares e Moreno (1994) afirmam que a redução da qualidade da água e de suas variáveis físico-químicas pode provocar o estresse e mortalidade de peixes, e segundo Proença e Bittencourt (1994), a morte dos animais é a resposta mais extrema a um fator estressante, mas antes ocorrem mudanças no comportamento, redução no desenvolvimento e na 
tolerância às enfermidades. Entre os fatores abióticos que mais influenciaram o desenvolvimento, a temperatura e o oxigênio dissolvido foram os principais.

Para a temperatura, todos os tratamentos apresentaram variações semelhantes, oscilando pela manhã, entre 25 e $28^{\circ} \mathrm{C}$, e à tarde entre 26 e $29^{\circ} \mathrm{C}$, excetuando-se o período do $13^{\circ}$ e $20^{\circ}$ dia de experimento, quando houve um decréscimo, chegando a $22,5^{\circ} \mathrm{C}$, pela manhã e $22,9^{\circ} \mathrm{C}$ a tarde no 17ㅇ dia. Lagler et al. (1977), Hepher (1988), Landau (1992) e Wootton (1992) afirmam que a temperatura tem grandes influências sobre a alimentação, metabolismo e desenvolvimento de peixes, isto também tem sido comprovado por Doria e Leonhardt (1993) e Ferraz de Lima et al. (1988), trabalhando com alevinos. Além de afetar o metabolismo de peixes, a temperatura exerce também uma grande influência nos processos fisiológicos dos organismos planctônicos (SOMMER, 1994).

Os resultados referentes à disponibilidade de oxigênio dissolvido (OD), variação de $\mathrm{pH}$ e valores de condutividade elétrica (Figura 2), apresentam o OD como um fator abiótico limitante agindo como fator estressante para os peixes, podendo ser responsável pelas mortes, devido seus baixos teores durante a madrugada. Sua disponibilidade depende de fatores como temperatura, salinidade, pressão atmosférica, decomposição de matéria orgânica e respiração dos organismos aquáticos (PROENÇA; BITTENCOURT, 1994). Observou-se uma queda brusca da disponibilidade inicial de OD em todos os tanques, devido a introdução dos peixes e sua respiração e pelo fato da baixa disponibilidade de plâncton (TAVARES; MORENO, 1994), evidenciado pelo tratamento SAO, cuja queda foi mais brusca, chegando a $0,7 \mathrm{mg} / \mathrm{L}$ no quarto dia. Boyd (1982) afirma que os efeitos da exposição de peixes a teores de OD abaixo de $1 \mathrm{mg} / \mathrm{L}$ por algumas horas pode causar a morte e exposições contínuas a teores entre 1 e $5 \mathrm{mg} / \mathrm{L}$ causam redução no crescimento, e recomenda teores acima de $5 \mathrm{mg} . \mathrm{L}^{-1}$ para o desenvolvimento normal dos peixes.

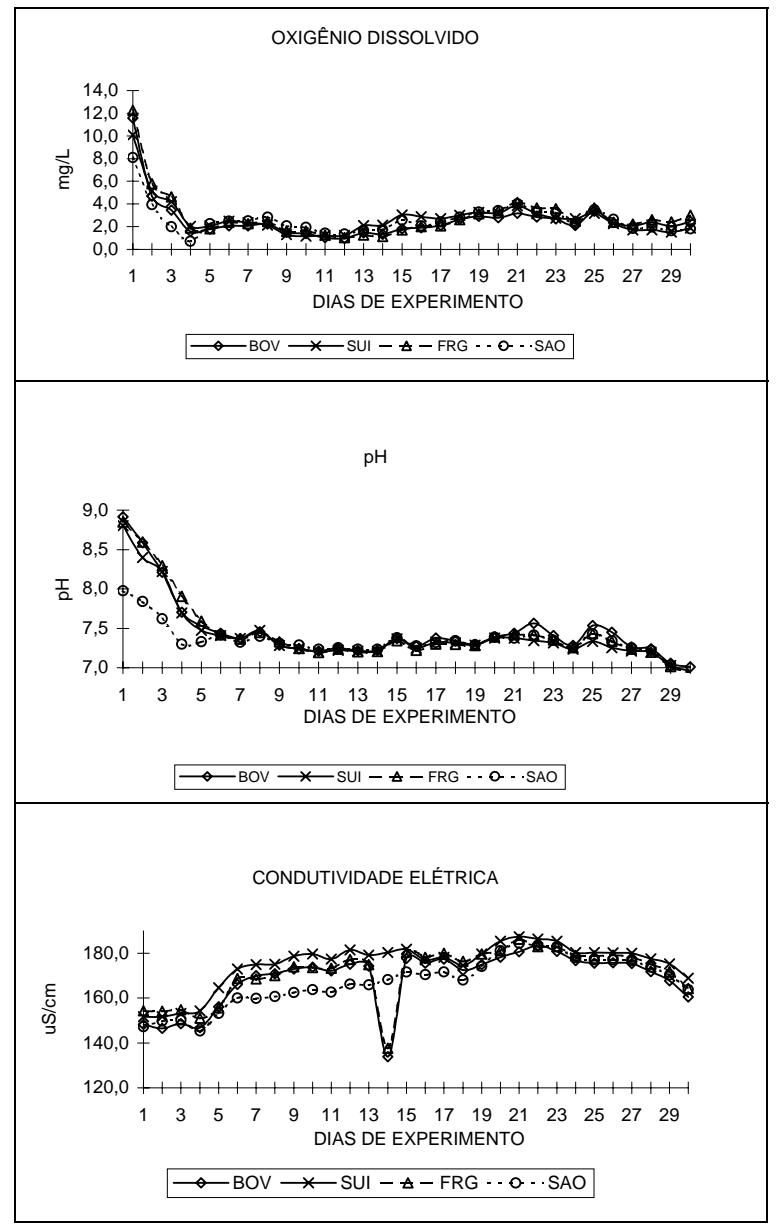

Figura 2. Valores médios do oxigênio dissolvido, $\mathrm{pH}$ e condutividade elétrica nos tratamentos experimentais.

Entre o 9 o e o $12^{\circ}$ dia, os teores estiveram abaixo de $2,0 \mathrm{mg} / \mathrm{L}$, e do $13^{\circ}$ e $20^{\circ}$ dia, quando houve uma queda acentuada da temperatura, a disponibilidade de OD teve um pequeno acréscimo, o que se deve a solubilidade do oxigênio (WETZEL, 1981). Os valores de $\mathrm{pH}$ permaneceram dentro da faixa recomendada por Boyd (1982), havendo um decréscimo inicial promovido pelo fornecimento de ração e a adição de estercos, aumentando a concentração de matéria orgânica dissolvida e acidificando o meio. A condutividade elétrica da água apresentou valores bastante elevados, entre 133,7 e 187,4 uS/cm. A condutividade aumentou após a introdução dos peixes devido a liberação de nutrientes para o meio (TAVARES; MORENO, 1994), e observaram-se valores maiores para os tratamentos adubados com 
estercos, o que está de acordo com Takino e Cipólli (1988). Segundo Tavares, Bachion e Rocha (1994), em ambientes eutróficos os valores de condutividade podem chegar a $240 \mathrm{uS} / \mathrm{cm}$, mas viveiros de piscicultura possuem valores menores pelo efeito diluidor do fluxo contínuo, curto tempo de residência da água, e menor acúmulo de íons. A disponibilidade de organismos planctônicos durante o experimento variou muito, tanto no aspecto quantitativo como qualitativo, o que influiu certamente no desempenho final dos diferentes tratamentos.

Os tratamentos tiveram um comportamento diferenciado em relação ao desenvolvimento planctônico, destacando-se os tratamento SUI e FRG até a metade do experimento, apresentando densidade fitoplanctônica de 300 mil indivíduos por litro no $12^{\circ}$ dia para SUI e 272 mil indivíduos por litro para FRG no $13^{\circ}$ dia. Os tratamentos BOV e SAO apresentaram uma baixa densidade inicial, tendo um aumento lento durante todo o período para o BOV, e um aumento a partir da segunda semana para o SAO. Isto pode ser explicado pelo fato de que a adubação orgânica eleva os níveis de nutrientes e aumenta a biomassa algal, sendo que no tratamento SAO este aumento somente ocorreu após a fertilização pelo arraçoamento e das excretas dos peixes (TAVARES; COLUS, 1995). Os grupos fitoplanctônicos mais representativos durante o período estudado (Figura 3), foram as Clorófitas e Cianófitas em todos os tratamentos, estando de acordo com por Ribeiro, Kubo e Mainardes-Pinto (1997).

Esta predominância deve-se a eutrofização do ambiente, que provocou um grande aumento na densidade destes grupos durante o período, destacando-se entre as algas verdes a classe Chlorophyceae, com o gênero Scenedesmus, e entre as cianofíceas destacou-se o gênero Microcystis. A alta densidade observada para estes grupos deve-se as condições ambientais favoráveis, como pH, temperatura e disponibilidade de nutrientes (ESTEVES, 1998).

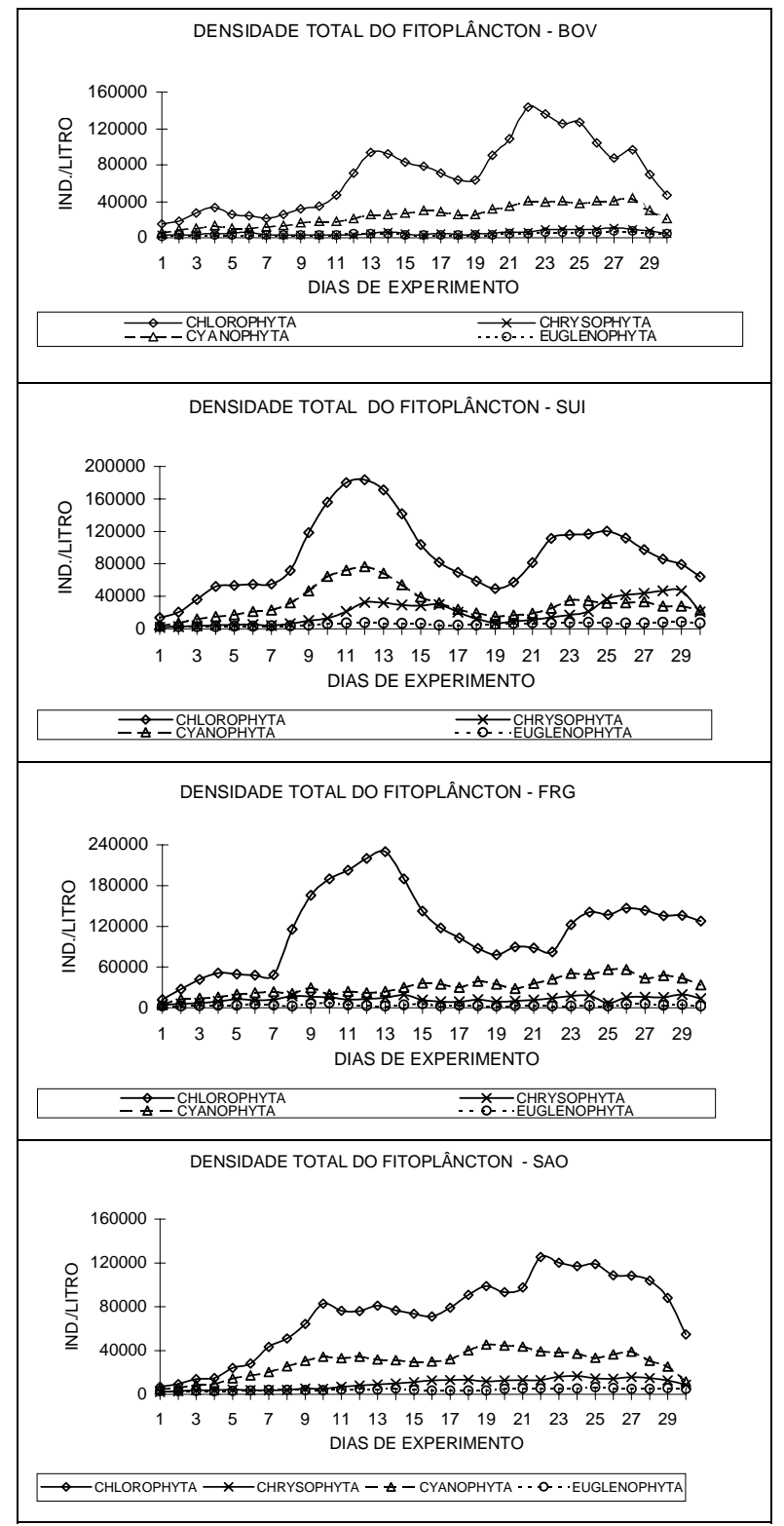

Figura 3. Valores médios dos diferentes grupos fitoplanctônicos nos tratamentos experimentais.

Entre os tratamentos destacaram-se o FRG e SUI, que apresentaram no final da segunda semana, os picos populacionais. Os grupos das Crisófitas e Euglenófitas foram menos abundantes, sendo que entre as Crisófitas a maior densidade foi de organismos da classe Bacillariophyceae. Estes grupos não apresentaram picos populacionais mas aumentaram a densidade durante todo o período. As Euglenófitas apresentaram maior densidade no período de maior eutrofização, e isto está de acordo 
com Xavier (1993), que afirma que estas possuem um alto grau de heterotrofia, vivendo em ambientes ricos em matéria orgânica.

Durante todo o experimento houve a predominância de organismos nanoplanctônicos de clorofíceas, cianofíceas e diatomáceas, favorecendo o desenvolvimento do zooplâncton, e desempenhando um papel importante na dieta alimentar dos peixes (ESKINAZI-LEÇA; KOENING, 1980).

A densidade do zooplâncton variou muito, com a maior densidade no tratamento FRG no $16^{\circ}$ dia de experimento, estando de acordo com Motokubo et al. (1988) e Faria (1997). Entre os grupos zooplanctônicos (rotíferos, copépodos e cladóceros), a maior abundância foi de rotíferos, confirmado por Soares et al. (1997), com destaque para Keratella. A alta densidade destes organismos deve-se ao seu ciclo de vida curto, possibilitando assim a rápida proliferação, quando em condições ambientais favoráveis, e a queda brusca da sua população após a diminuição do fitoplâncton, ocorrida na terceira semana, deve-se a associação acentuada dos rotíferos ao fitoplâncton pela predação (ESTEVES, 1998). Os copépodas foram o segundo grupo mais numeroso sendo representado por copépodas ciclopóides, na maioria náuplios e copepoditos, ocorrendo poucos adultos. Como o ciclo de vida destes organismos é mais longo e a possibilidade de predação pelos peixes maior devido ao seu maior tamanho, houve um aumento da densidade apenas na segunda metade do experimento, destacando-se o tratamento BOV. Os cladóceros foram encontrados em baixa densidade em todos os tratamentos, o que pode ter sido influenciado pelos fatores abióticos, como a temperatura, que influencia muito a reprodução, pelo seu tamanho e pela predação sofrida. Segundo Tavares, Bachion e Rocha (1994), os cladóceros são mais vulneráveis à predação do que os copépodas, devido ao mecanismo de captura por sucção dos peixes, e pelo fato de não apresentarem habilidade de escapar como os copépodas.

\section{Conclusão}

Podemos concluir que nas condições do experimento, o tratamento adubado com esterco de frangos possibilitou um maior desenvolvimento da comunidade planctônica, principalmente de organismos-alimento, e melhores resultados quanto ao desenvolvimento dos peixes, demonstrando a importância do alimento natural na sua dieta.

\section{Referências}

AGOSTINHO, A. A.; ZALEWSKI, M. A planície alagável do Alto Rio Paraná. Maringá: Ed. UEM, 1996.

BONECKER, C. C.; LANSAC-TÔHA, F. A.; STAUB, A. Qualitative study of rotifers in different enviroments of the high Paraná River floodplain (MS) - Brazil. Revista Unimar, Maringá, v.16, n.3, p.1-16, 1994.

BORGHETTI, J. R.; CANZI, C.; NOGUEIRA, S. V. G. A influência da proteína no crescimento do matrinchã (Brycon orbignyanus) criado em tanque-rede. Revista Brasileira de Biologia, Rio de janeiro, v.51, n.3, p.695-699, 1991.

BOYD, C. E. Water quality management for pond fish culture. Amsterdam: Elsevier Scientific Publishing, 1982.

BRITSKI, H. A.; SATO, Y.; ROSA, A. B. Manual de identificação de peixes da bacia do São Francisco. 3.ed. Brasília: CODEVASF, 1988.

CECCARELLI, P. S.; SENHORINI, J. A. Brycon: Viabilização da produção de alevinos. Panorama da Aqüicultura, Rio de Janeiro, v.6, n.35, p.10-11, 1996.

COMPANHIA DE TECNOLOGIA DE SANEAMENTO AMBIENTAL. Piscicultura e qualidade das águas no Estado de São Paulo. São Paulo, 1982. v.1

CONTE, L.; BOZANO, G. L. N.; FERRAZ DE LIMA, J. A. Influência do sistema de alimentação no crescimento da Piracanjuba Brycon orbignyanus, em gaiolas. Boletim Técnico CEPTA, Pirassununga, n.8, p.49-59, 1995.

DORIA, C. R. C.; CAVICCHIOLI, M.; ZANONI, M. A.; LEONHARDT, J. H. Análise preliminar da produção do policultivo semi-intensivo com arraçoamento e adubação orgânica de Cyprinus carpio (Pisces:Cyprinidae), Prochilodus scrofa (Pisces: Prochilodontidae), Piaractus mesopotamicus e Colossoma macropomum (Pisces: Characidae). Revista Unimar, Maringá, v.15, n.1, suplemento, p.233-242, 1993. 
DORIA, C. R. C.; LEONHARDT, J. H. Análise do crescimento de Piaractus mesopotamicus e Colossoma macropomum (Pisces: Characidae) em sistema semiintensivo de policultivo com arraçoamento e adubação orgânica. Revista Unimar, Maringá, v.15, n.1, suplemento, p.211-222, 1993.

ESKINAZI-LEÇA, E.; KOENING, M. Composição do fitoplâncton dos viveiros de criação de peixes da região de Itamaracá (PE). In: SIMPÓSIO BRASILEIRO DE AQUICULTURA, 1., 1978, Recife. Anais... Rio de Janeiro: Academia Brasileira de Ciências, 1980. p. 87-97.

ESTEVES, F. A. Fundamentos de Limnologia. 2.ed. Rio de Janeiro: Interciência, 1998.

FARIA, A. C. E. A. Produção de organismos-alimento em tanques experimentais submetidos a diferentes adubações orgânicas.1997. Trabalho de Conclusão de Curso (Bacharel em Ciências Biológicas)- Universidade Estadual de Maringá, Maringá.

FERRARI, V. A.; LUCAS, A. F. B.; GASPAR, L. A. Monocultura de pacu Piaractus mesopotamicus (HOLMBERG, 1887) em condições de viveiro-estufa.. Boletim Técnico CEPTA, Pirassununga, v.3, p.33-42. 1991.

FERRAZ DE LIMA, J. A.; FERRARI, V. A.; COLARES DE MELO, J. S.; GASPAR, L. A.; CHABALIM, E.; SANTOS, E. P. Comportamento do Pacu Colossoma mitrei, em um cultivo experimental, no Centro-Oeste do Brasil. Boletim Técnico CEPTA, Pirassununga, v.1, n.1, p.15-28. 1988.

HEPHER, B. Nutrition of pond fishes. In: HUET, M. 1978 Tratado de piscicultura. 2ed., Madrid: Mundi Prensa,. 1988.

HUSZAR, V. L. M.; WERNECK, A. M.; ESTEVES, F. A. Dinâmica nictemeral da comunidade fitoplanctônica em relação aos principais fatores abióticos na lagoa Juparanã, Linhares, Espirito Santo, Brasil: Fevereiro de 1987. Revista Brasileira de Biologia, Rio de janeiro, v.54, n.1, p.111134, 1994.

KOEHLER, H.S. Estatística experimental. Curitiba: Ed. UFPR, 1994.

LAGLER, K. F.; BARDACH, J. E.; MILLER, R. R.; PASSINO, D. R. M. Ichthyology. 2.ed. New York: John Willey, 1977.

LANDAU, M. Introduction to aquaculture. New York: John Willey, 1992.

LEWIS Jr., W. M. Zooplancton community analysis: studies on a tropical system. New York: Springer Verlag, 1979.

MATSUMURA-TUNDISI, T; ROCHA, O. Occurence of copepod (Calanoida Cyclopoida and Harpacticoida) from "Broa" Reservoir (São carlos, São Paulo, Brazil). Revista Brasileira de Biologia, Rio de Janeiro, v.43, n.1, p.1-17, 1983.
MENDONÇA, J. O. J. Criação de espécies do gênero Brycon no CEPTA/IBAMA. In: SEMINÁRIO SOBRE A CRIAÇÃODE ESPÉCIES DO GÊNERO BRYCON, 1., 1994, Pirassununga. Anais... Pirassununga: CEPTA, 1994. p.31.

MENDONÇA, J. O. J. O gênero Brycon. Panorama da Aqüicultura, Rio de Janeiro, v.6, n.33, p.14-16, 1996.

MOTOKUBO, M. T.; ANTONIUTTI, D. M.; MAINARDES-PINTO, C. S. R.; TAKINO, M. Produção de zoopâncton em tanques de cultivo de curimbatá, Prochilodus scrofa, submetidos a diferentes fertilizantes orgânicos. Boletim do Instituto de Pesca, São Paulo, v.15, n.2, p.189-199, 1988.

NAKATANI, K.; AGOSTINHO, A. A.; BAUMGARTNER, G.; BIALETZKI, A.; SANCHES, P. V.; MAKRAKIS, M. C.; PAVANELLI, C. S. Ovos e larvas de peixes de água doce: desenvolvimento e maual de identificação. Maringá: EDUEM, 2001. v. 1.

NEEDHAM, P. R.; NEEDHAM, J. G. Guia para el estudio de los seres vivos de las aguas dulces. Barcelona: Reverté, 1982.

OLIVEIRA E SILVA, S. L.; COELHO, J. B.; DIAS, J. R. C.; PINTO, J. B.; CRISÓSTOMO, L. C. Cultivo de peixes em ambientes fertilizados com efluente de biodigestor. In: SIMPÓSIO BRASILEIRO DE AQÜICULTURA, 3, 1983, São Carlos. Anais... São Carlos: Associação Brasileira de Aqüicultura, 1984. p.165-185.

OLIVEIRA, M. D.; TRAIN, S.; RODRIGUES, L. C. Levantamento preliminar do fitoplâncton de rede (exceto Zignemaphyceae) do rio Paraná, no município de Porto Rico, Paraná, Brasil. Revista Unimar, Maringá, v.16, n.3, p.155-174, 1994.

PAIVA, M. P. Peixes e pescas de águas interiores do Brasil. Brasília: Editerra, 1983.

PARANAGUÁ, M. N.; KOENING, M. L. Composição e "Standing-stock" do zooplâncton dos viveiros de criação de peixes da região de Itamaracá (PE). In: SIMPÓSIO BRASILEIRODE AQUICULTURA, 1, 1978, Recife. Anais... Rio de Janeiro: Academia Brasileira de Ciências, 1980. p. 99-107.

PICELLI-VICENTIM, M. M. Chlorococcales planctônicas do Parque Regional do Iguaçú, Curitiba, Estado do Paraná. Revista Brasileira de Biologia, Rio de janeiro, v.47, n.1/2, p.57-85.

PIMENTEL, F. G. Curso de Estatística(Esalq/USP). 12. ed. Piracicaba: Nobel, 1987.

PROENÇA, C. E. M.; BITENCOURT, P. R. L. Manual de piscicultura tropical. Brasília: IBAMA, 1994.

RIBEIRO, M. A. G.; KUBO. E.; MAINARDES-PINTO, C. S. R. Efeito do adubo orgânico e da dosagem do fertilizante químico no aumento do fitoplâncton e do zooplâncton. Boletim do Instituto de Pesca, São Paulo, v.24, p.57-64, 1997. 
SENDACZ, S.; KUBO, E. Copepoda (Calanoida e Cyclopoida) de reservatórios do Estado de São Paulo. Boletim do Instituto de Pesca, São Paulo, v.9, p.51-89, 1982.

SENHORINI, J. A.; FIGUEIREDO, G. M.; FONTES, N. A.; CAROLSFELD, J. Larvicultura e alevinagem do pacu Piaractus mesopotamicus (HOLMBERG,1887), tambaqui Colossoma macropomum (CUVIER,1818) e seus respectivos híbridos. Boletim Técnico CEPTA, Pirassununga, v.1, n.2, p.19-30, 1988.

SOARES, C. M.; HAYASHI, C.; FURUYA, W. M.; FURUYA, V. R. B.; MARANHÃO, T. C. F. Alimentação natural de larvas do cascudo preto Rhinelepis aspera AGASSIS, 1829 (Osteichthyes - Loricariidae) em tanques de cultivo. Boletim do Instituto de Pesca, São Paulo, v.24 , n.esp., p.109-117, 1997.

SOMMER, U. Planktologie. Heidelberg, Springer Verlag, In: STEFFENS, W. Princípios fundamentales de la alimentación de los peces. Barcelona: Acribia, 1994.

STEFFENS, W. Principios fundamentales de la alimentación de los peces. Barcelona: Acribia, 1987.

TAKINO, M.; CIPÓLLI, M. N. Caracterização limnológica em tanques de cultivo de tilápia, Oreochromis niloticus: parâmetros físicos, químicos e clorofila $a$. Boletim do Instituto de Pesca, São Paulo, v.15, n.2, p.237-245, 1988.

TAVARES, L. H. S.; COLUS, D. S. O. Estudo da variação nictemeral em um viveiro de piscicultura no período de seca. Revista Unimar, Maringá, v.17, n.2, p.225-236, 1995.
TAVARES, L. H. S.; MORENO, S. Q. Variação dos parâmetros limnológicos em um viveiro de piscicultura nos períodos de seca e chuva. Revista Unimar, Maringá, v.16, n.4,p.229-242, 1994.

TAVARES, L. H. S; BACHION, M. A.; ROCHA, O. Estudo do crescimento populacional de três espécies zooplanctônicas em laboratório e o uso do plâncton na alimentação de alevinos de Oreochromis niloticus (tilápia) e Astyanax scabripinis paranae (Lambari). Revista Unimar, Maringá, v.16, n.3, p.189-201,1994.

UNIVERSIDADE ESTADUAL DE MARINGÁ. Relatório de Estudos limnológicos e ictiológicos em áreas de reprodução coletiva de peixes do rio Paraná, nas imediações do município de Porto Rico - Outubro de 1986 a Março de 1987. Maringa: Fundação Universidade Estadual de Maringá, 1989.

Relatório de Estudos ambientais na planície de inundação do rio Paraná no trecho compreendido entre a foz do rio Paranapanema e o reservatório de Itaipú. Maringa: Fundação Universidade Estadual de Maringá/ NUPELIA, 1993

WETZEL, R. G. Limnología. Barcelona: Omega, 1981.

WOOTTON, R. J. Ecology of teleost fishes. London: Chapman \& Hall, 1992.

XAVIER, M. B. Distribuição vertical das Euglenaceae pigmentadas do rio Grande, Represa Billings, São Paulo, Brasil. Acta Limnologica Brasiliensia, São carlos, v.6, n.1, p.11-30, 1993. 